\title{
The Effects of Physical Filtration on the Control of Microcystis aeruginosa at Various Growth Stages
}

\author{
Tao Song ${ }^{4,5}$, Xiangen $X u^{1,2} \#$, Ligang $X u^{1,3 *}$ \\ 'Key Laboratory of Watershed Geographic Sciences, Nanjing Institute of Geography and Limnology, \\ Chinese Academy of Sciences, Nanjing 210008, China \\ ${ }^{2}$ Changzhou Environmental Science Research Institute, Changzhou 213022, China \\ ${ }^{3}$ Eco-Environmental Engineering Research Center, China Three Gorges Corporation, Beijing 100038, China \\ ${ }^{4}$ Institute of Karst Geology, CAGS/Key Laboratory of Karst Dynamics, \\ Ministry of Natural Resources \& Guangxi, Guilin 541004, China \\ ${ }^{5}$ China University of Geosciences Beijing, Beijing 100083, China
}

Received: 8 March 2021

Accepted: 1 June 2021

\begin{abstract}
Physical filtration technique has been widely used throughout the world as a control for cyanobacteria. However, few studies have addressed the time and strength of the filtration making it a challenge to provide effective support for the filtration of cyanobacteria. Therefore, it is urgent to study the control feedback effect of cyanobacteria after physical filtration with different control intensities. In this study, through laboratory simulation experiments, the growth feedback rule of Microcystis aeruginosa, a dominant species of cyanobacteria in Lake Tai Basin, was systematically studied following different intensity physical filtration at various growth stages. Based on the results, the optimal control period and control intensity of cyanobacteria were proposed. The results showed that the growth of Microcystis aeruginosa conforms to the Logistic growth model. Physical filtration in the early and late stages of the Logistic start period was effective in inhibiting Microcystis aeruginosa, while filtration and inhibition in the early and middle stages of Logistic accelerated period were not effective. The growth of Microcystis aeruginosa was affected by different control intensity at different stages and can be effectively inhibited by $20-40 \%$ controlled intensity filtration in the early and late stages of the Logistic start period. If cost and environmental benefits are considered, we recommend the implementation of physical filtration at a minimum of $20 \%$ control intensity at the beginning of the Logistic start period.
\end{abstract}

Keywords: physical filtration, Microcystis aeruginosa, growth stages, logistic model, cyanobacteria bloom control

\footnotetext{
\#These authors contributed equally to the work.

*e-mail: $\lg x u @$ niglas.ac.cn
} 


\section{Introduction}

With the rapid development of the economy and the expansion of human activity, a large amount of nitrogen, phosphorus, and other elements are discharged into the water, resulting in the eutrophication of lakes and other bodies of water, leading to the proliferation of cyanobacteria [1-2]. The proliferation and abnormal growth of cyanobacteria form blooms and cause algal populations to accumulate, rot, and release algal toxins onto lakeshores and into estuaries [3-4]. This can reduce the efficiency of water resource utilization, seriously damage the ecological balance and water landscape of lakes, and cause significant economic losses [5-8]. Therefore, effective control of the explosive growth of algae has become an important issue and requires an effective solution to control lake eutrophication and restore aquatic ecosystems.

The current control methods of cyanobacteria bloom primarily include chemical, biological, and physical methods [9-10]. Among them, the chemical methods will cause serious harm to the lake ecosystem, the biological methods is slow to take effect [11-14], the investment is large, and the physical method is pollution-free and effective [15-18], so physical method is the most widely used method. The main physical methods can include: Cyanobacteria salvage, sand filling, nutrient salt passivation, bottom aeration, dilution and erosion, bottom sediment covering, flocculation and settlement [19], etc. As for the control of cyanobacteria bloom, it is necessary to choose different control methods or combinations of different control methods according to different scenarios. For example, sand filling, nutrient salt passage, bottom aviation, diffusion and erosion, bottom division covering are suitable for the long-term control of cyanobacteria bloom. Cyanobacteria salvage, flocculation and settlement are suitable for emergency control of cyanobacteria bloom. Compared with other control methods, cyanobacteria salvage has the advantages of a quick effect and no secondary pollution [20-21], and can reduce the nitrogen and phosphorus load in the water body within a certain range, and is gradually becoming a significant means to control cyanobacteria bloom.

Cyanobacteria salvage is widely used in an emergency to control cyanobacteria accumulation pollution, and more and more researchers began to pay attention to the dynamics of cyanobacteria control. Zhou [11] showed that cyanobacterial salvage has a certain reduction effect on nitrogen and phosphorus nutrients in the water column and cyanobacterial salvage has a significant effect on controlling cyanobacterial density based on indoor and outdoor grid tests. Chen [20] investigated the effectiveness of cyanobacterial salvage in reducing cyanobacterial density and controlling cyanobacterial outbreaks based on indoor and outdoor grid tests, respectively, and the effectiveness of cyanobacterial salvage in controlling nutrient levels in the water column. Although cyanobacteria salvage methods have received more attention in recent years, there are few studies on the growth feedback effect of cyanobacteria after physical filtration at various growth stages.

Microcystis aeruginosa is the dominant species of summer bloom cyanobacteria in Lake Tai, the third largest freshwater lake in China, and dominates the competition among different species of bloom cyanobacteria. In order to better grasp the growth law of cyanobacteria after salvage, find the best control intensity and control period of the cyanobacteria salvage project, so as to improve the efficiency of cyanobacteria salvage in Lake Tai. Therefore, the growth feedback of Microcystis aeruginosa at different growth stages and various filtration intensities were studied. Moreover, the best growth period and filtration intensity for Microcystis aeruginosa were selected. This study provides a scientific basis for the selection of the best salvage time for cyanobacteria and theoretical support for the exploration of the control method of cyanobacteria populations.

\section{Experimental}

\section{Experimental Material}

Single-celled Microcystis aeruginosa (PCC 7806) was purchased from the Institute of Hydrobiology, Chinese Academy of Sciences. The BG11 medium was used for inoculation in a sterile environment and the algal solution was cultured in an incubator (LRH-150). The culture conditions were as follows: the temperature was $25 \pm 1^{\circ} \mathrm{C}$, light intensity was $30 \mu \mathrm{mol} \mathrm{m} \mathrm{m}^{-2} \cdot \mathrm{s}^{-1}$, and the light-dark cycle was 12:12 hours (L:D). The glassware for the experiment was washed with clean water, soaked in $0.1 \mathrm{~mol} / \mathrm{L}$ diluted hydrochloric acid, washed with sterile water, dried, and autoclaved $(0.15 \mathrm{MPa}$ and $120^{\circ} \mathrm{C}$ ) for later use.

\section{Experimental Conditions}

A pre-experimental group or control and four treatment groups were set up, denoted as the control group and treatment groups A, B, C, and D, respectively. Three parallel groups were set for each group. Treatment groups A, B, C, and D were determined based on the Logistic growth period of Microcystis aeruginosa used in the experiment. Three control intensities were set for each treatment: $20 \%$ filter (T20), 40\% filter (T40) and $60 \%$ filter (T60).

The mother liquor of M. aeruginosa was inoculated into $400 \mathrm{ml}$ of algal fluid in a $500 \mathrm{ml}$ Erlenmeyer flask on a super-clean workbench. The initial absorbance of inoculated algal fluid was 0.18 , determined at $680 \mathrm{~nm}$ using an ultraviolet spectrophotometer (UV-1800). The culture was sealed with sealing film and grown in an incubator. The Erlenmeyer flask oscillated twice a day at a fixed time, and the first day of inoculation 


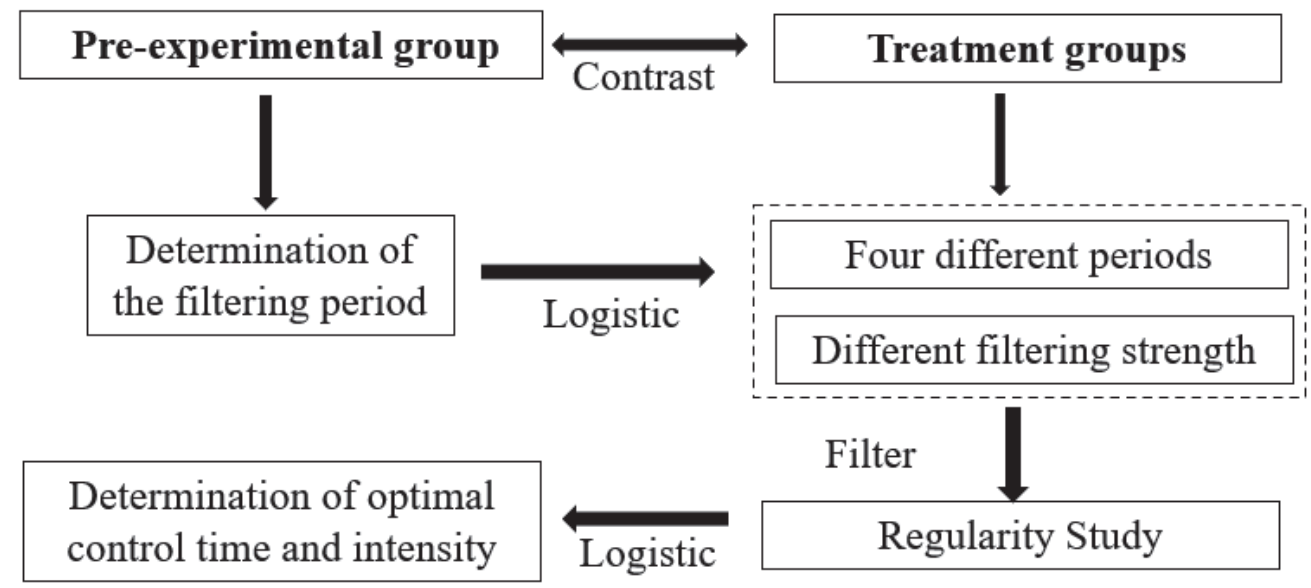

Fig. 1. Experimental flow.

was recorded as the culture day 0. Treatment group A was filtered with a $0.45 \mathrm{um}$ acetate fiber membrane on the second day after inoculation, and the filtrate was transferred into the Microcystis aeruginosa stock solution. The filtration day of treatment group A was recorded as the first culture day.

The experimental flow chart is as follows (Fig. 1).

The growth of Microcystis aeruginosa was fitted by the Logistic model using the following equation [22-24]:

$$
y=\frac{A_{1}-A_{2}}{1+\left(x+x_{0}\right)^{p}}+A 2
$$

In order to investigate the $\mathrm{k}$ value and growth patterns of Microcystis aeruginosa, the experiment designed the growth pre-experiment of Microcystis aeruginosa in the same batch. The results showed that the growth absorbance limit of Microcystis aeruginosa in the laboratory was about 2.0 and the growth was consistent with Logistic growth. Based on the preexperimental results, the A2 value of the Logistic model used in this experiment was set as fixed value 2. The

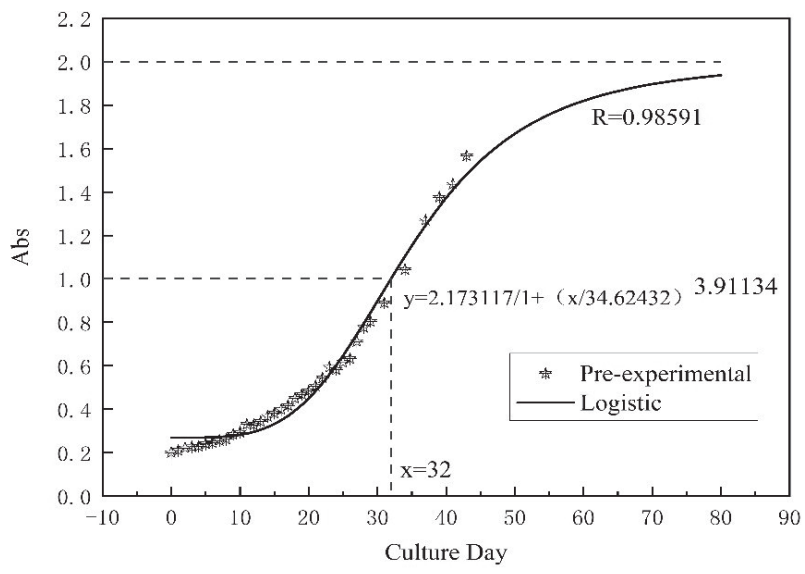

Fig. 2. Logistic fitting of growth of Microcystis aeruginosa in the pre-experiment.
Logistic model (fitting performed with Orgin 2019) was used to fit the model of the same batch of Microcystis aeruginosa in the pre-experiment.

According to the fitting results, $\mathrm{R}=0.98591$, $\mathrm{y}=2.173117 / 1+(\mathrm{x} / 34.6232) 3.91134$, and the fitting degree were high. The growth of Microcystis aeruginosa in the pre-experiment was consistent with Logistic growth. Based on the actual growth conditions and Logistic growth model of the Microcystis aeruginosa in the pre-experiment, four physical filtrations were performed on the $1^{\text {st }}, 10^{\text {th }}, 19^{\text {th }}$, and $25^{\text {th }}$ day after inoculation. The first (A), second (B), third (C), and fourth physical filtrations (D) are respectively recorded below.

In this study, based on the $\mathrm{K}$ value and $\mathrm{K} / 2$ value in the Logistic fitting of the pre-experimental group, the period of the four physical filtering was defined. The results are as follows:

On the first day of incubation, Microcystis aeruginosa was in the early stages of the Logistic start period. The concentration and the growth rate Microcystis aeruginosa were low and the culture medium was full of nutrition. For the actual cyanobacteria salvage project, the concentration of cyanobacteria during this period was low and the salvage difficulty was small. Therefore, it was a suitable time to implement the first physical filtering. On the $10^{\text {th }}$ day of the incubation period, Microcystis aeruginosa was in the late stages of the Logistic start period. As the growth rate of Microcystis aeruginosa accelerated, the absorbance value reached 0.30 . During this time, the ability of $M$. aeruginosa to utilize nutrients was enhanced, as it had fully adapted to the new living environment after inoculation and thus the second physical filtration was done. On the $19^{\text {th }}$ day of the incubation period, $M$. aeruginosa was in the early stage of the logistic accelerated growth period. The growth rate of $M$. aeruginosa was further accelerated and the absorbance value reached approximately 0.4 On the $25^{\text {th }}$ day of the incubation period, M. aeruginosa was in the middle and later stages of the Logistic accelerated 


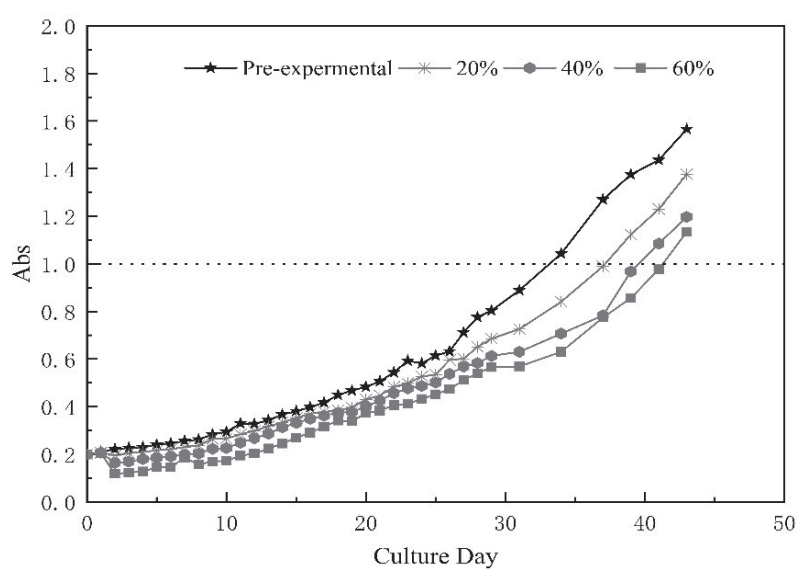

Fig. 3. The growth curve of Microcystis aeruginosa after physical filtration.

growth period, at which time the absorbance value of M. aeruginosa reached approximately 0.6 .

\section{Monitoring Index}

The absorbance of Microcystis aeruginosa was monitored daily during the experiment for 43 days, with samples collected every morning. The absorbance was determined at $680 \mathrm{~nm}$ using an ultraviolet spectrophotometer (UV-1800).
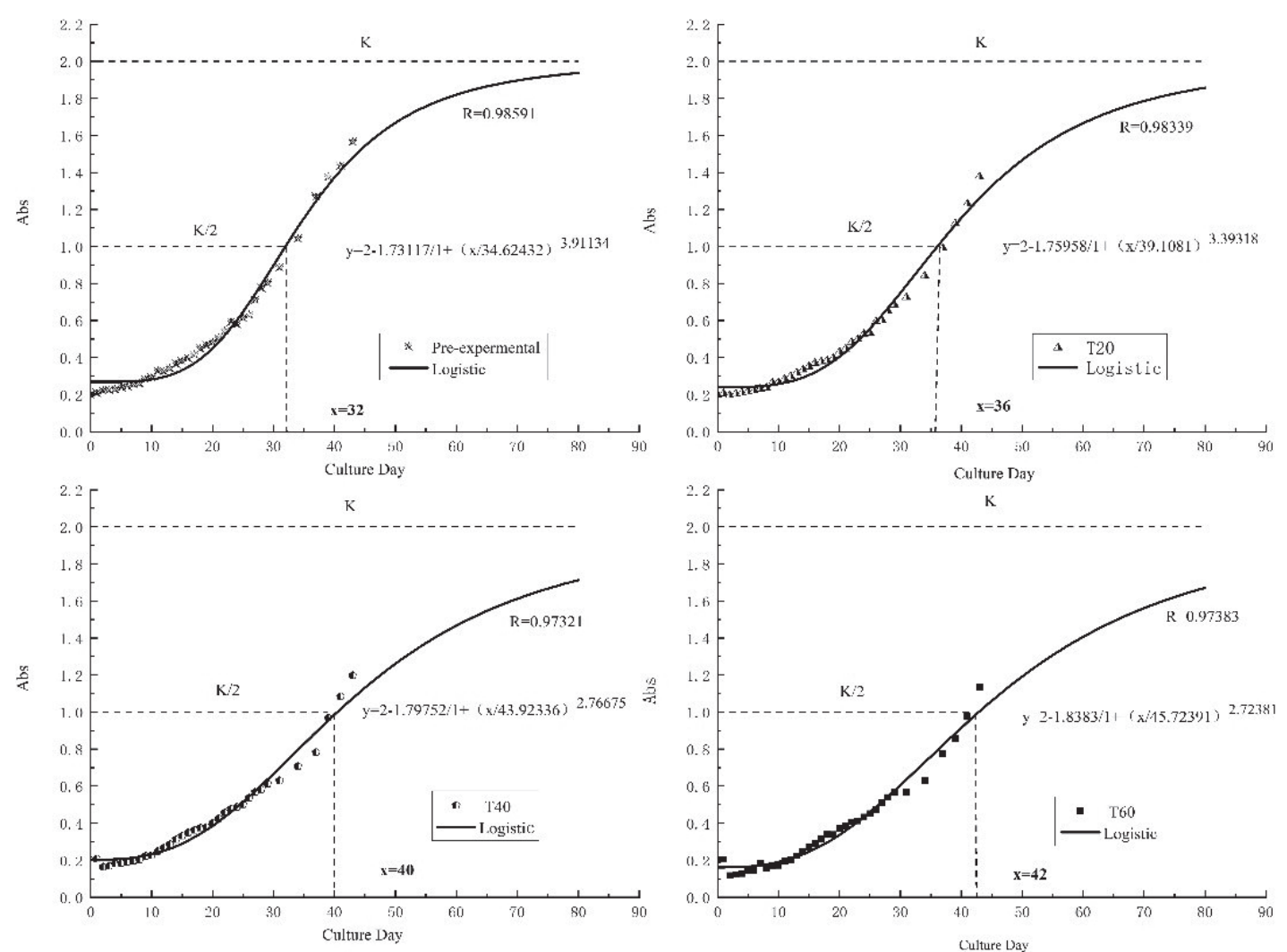

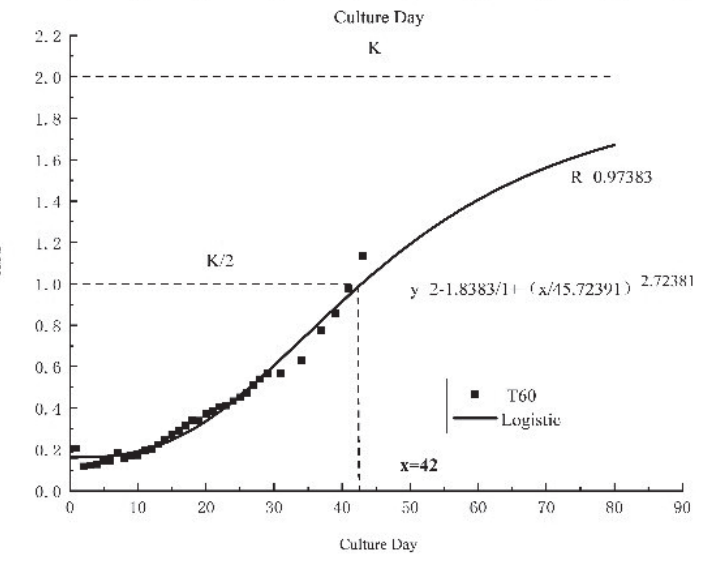

Data were processed using Excel 2010, and related image plotting and Logistic model fitting were performed with Orgin 2019.

\section{Results and Discussion}

Filtering in the Early Stages of the Logistic Start Period

Following physical filtration, the absorbance of Microcystis aeruginosa with different control intensities decreased to various degrees, where $60 \%>40 \%>20 \%$ (Fig. 3). The growth trend of the absorbance of Microcystis aeruginosa after filtration was consistent with that of the pre-experimental group. However, the absorbance after filtration was lower than that of the pre-experimental group. The absorbance of the filtration group was close to that of the pre-experimental group at 43 days.

The experiment showed that the limit absorbance value of Microcystis aeruginosa could reach 2.0 following inoculation. Therefore, the limit growth $\mathrm{A}_{2}$ (K value) in the Logistic regression equation was set to 2.0. The Logistic model was then used to fit the growth of Microcystis aeruginosa after physical filtration (Fig. 4). The resulting $\mathrm{R}$ values of the pre-experimental group, T20, T40, and T60 were 0.98591, 0.98339,

Fig. 4. Logistic fitting model of physical filtration. 
0.97321, and 0.97383, respectively. The fitting degree was relatively high, which could effectively simulate the subsequent growth patterns of Microcystis aeruginosa. The fitting curve showed that the incubation time of the pre-experimental group and the T20, T40, and T60 groups to reach the proliferation rate point $(\mathrm{K} / 2)$ was 32, 36, 40, and 42 days, respectively. After physical filtration, Microcystis aeruginosa reached the saturation density point $(\mathrm{K})$ and the maximum proliferation rate point $(K / 2)$ slower than the pre-experimental group without physical filtration. Moreover, with increased filtration intensity, the saturation density point $(\mathrm{K})$ and the maximum proliferation rate point $(\mathrm{K} / 2)$ declined, indicating that the physical filtration had a significant inhibitory effect on the growth of Microcystis aeruginosa.

\section{Analysis of Physical Filtration}

In laboratory conditions, the physical filtration of Microcystis aeruginosa at low concentrations was effective. Moreover, with increased control intensity, the control was more effective. Although cyanobacteria blooms in the natural environment will have variability in the amount of available nutrients, temperature, and light conditions [25-26], the filtration of cyanobacteria bloom in the early stage of formation in the field can effectively inhibit their growth. Finally, physical filtration can delay the risk of cyanobacteria blooms.

\section{Flitering in the Late Stages} of the Logistic Start Period

After 10 days of culture, Microcystis aeruginosa was fully adapted to the growing environment. The ability to absorb and utilize nutrients was enhanced, the growth rate accelerated, and it began to proliferate more rapidly. Here, the second physical filtration was carried out in the time point of rapid proliferation (Fig. 5).

Following filtration, the absorbance of Microcystis aeruginosa decreased in proportion to the filtration intensity (Fig. 5). But as the growth of the culture time, the growth curves of the algal fluids at the three control intensities essentially overlapped. During the incubation period from 10 to 20 days, the absorbance of Microcystis aeruginosa under T20 was still higher than that of T40 and T60. However, after 20 days, the absorbance values of Microcystis aeruginosa under three control intensities were similar, suggesting that as the control intensity of filtration increases at this stage as does the proliferation rate of Microcystis aeruginosa.

The $\mathrm{R}$ values of the pre-experimental group, T20, T40, and T60 were 0.98591, 0.98457, 0.97715, and 0.96963 , and had a high fitting degree to the Logistic model $(\mathrm{A} 2=2)$ (Fig. 6). Following physical filtration, the absorbance of the T20, T40, and T60 groups reached the maximum $\mathrm{K} / 2$ point at 40,39 , and 41 days, respectively. The Microcystis aeruginosa with different control intensity reached the maximum proliferation

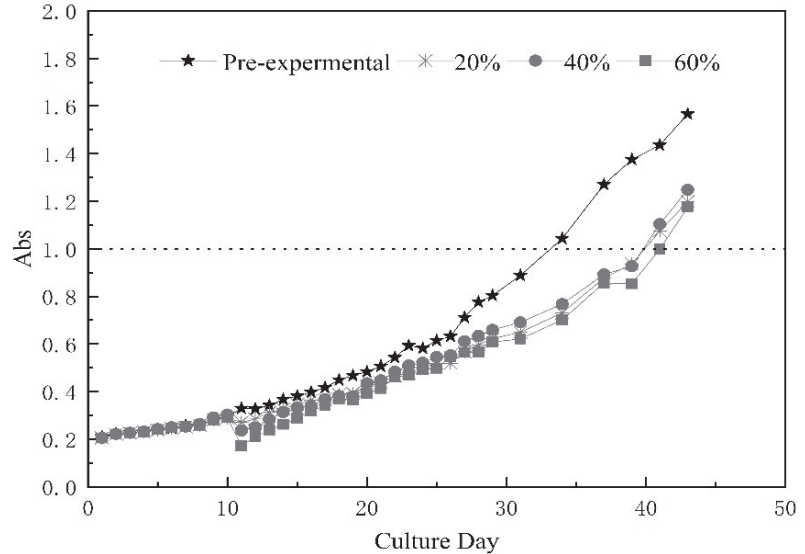

Fig. 5. The growth curve of Microcystis aeruginosa after physical filtration.

rate point at the same time point. This demonstrated that $M$. aeruginosa proliferation was effectively inhibited following the filtration on the 10th day. Additionally, with the increase of control intensity, the proliferation of Microcystis aeruginosa also increased due to several factors.

\section{Analysis of Physical Filtration}

During this filtration, Microcystis aeruginosa was in the stage of rapid proliferation. After the filtration, Microcystis aeruginosa concentration and the intraspecies competition were reduced. After the decrease of intra-species competition, the utilization efficiency of the nutrients and oxygen in the remaining culture was increased and the proliferation rate of the cyanobacteria was improved. As a result, the proliferation rate of Microcystis aeruginosa in group T60 was higher than that of group T40 or T20. This observation conforms to the Yoda et al. self-thinning $-2 / 3$ power law [2730], and the proliferation of Microcystis aeruginosa was treated as a self-thinning in group T60. When the population density reached a certain amount, the environmental carrying capacity $(\mathrm{K})$ was also reached. The larger intra-species competition did not only affect the population growth rate but also its survival. At this stage, the population was self-thinning, with increased survival of individuals that were larger and had higher vitality. These surviving individuals had a strong competitive ability and made full use of nutrients and light in the surrounding environment to maintain their rapid proliferation

In a practical cyanobacteria salvage project, the cyanobacteria salvage at this stage has a certain effect. However, during the period when the growth rate of algae was beginning to accelerate and when the control intensity was hight, the proliferation rate of cyanobacteria was higher, even reaching the density of algae under small control intensity. With the increase of salvage intensity, the cost also increases but there 

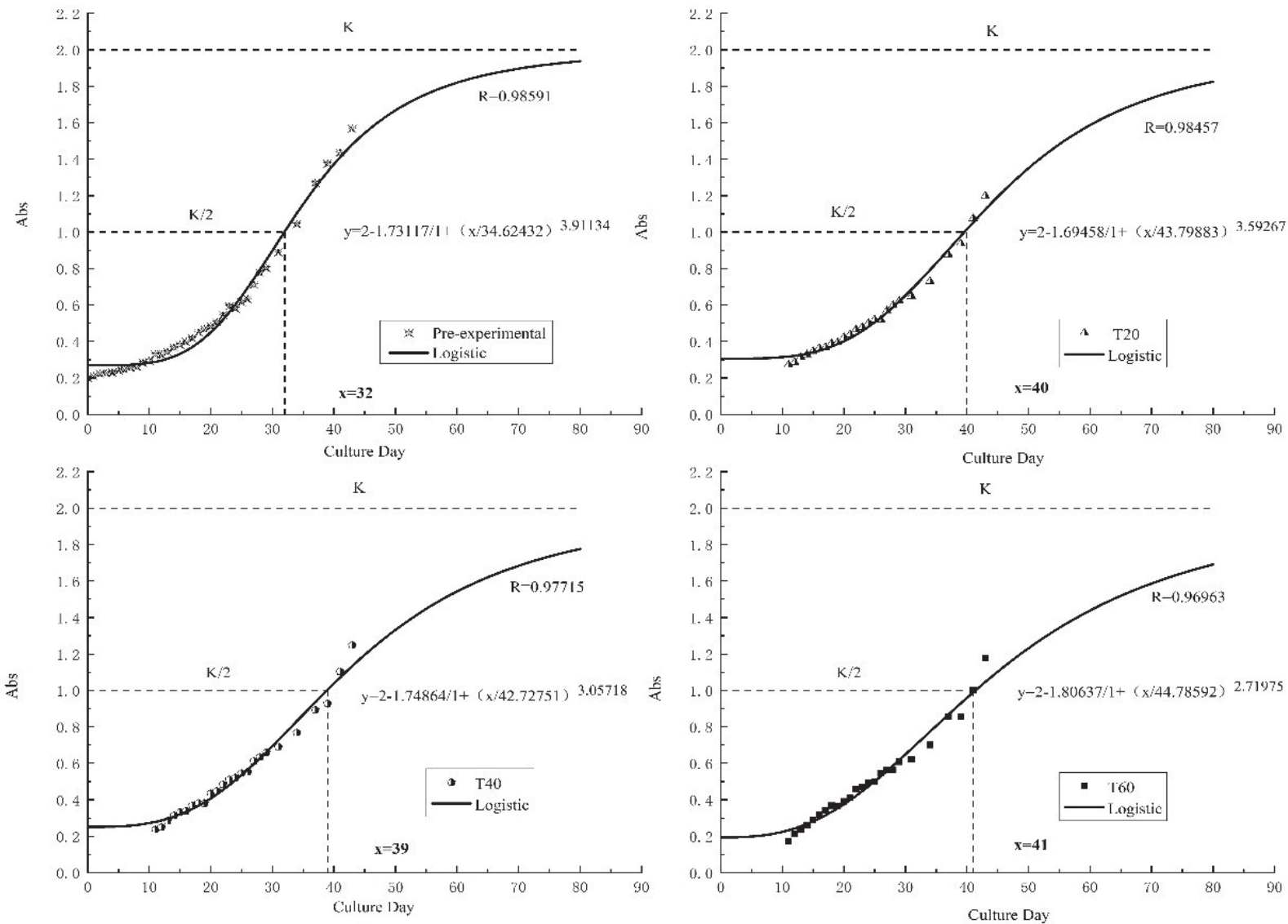

Fig. 6. Logistic fitting model of the second physical filtration.

are no added benefits. It is therefore best to choose a control intensity of about $20 \%$ at this stage.

\section{Filtering in the Early Stage of the Logistic Accelerated Growth Period}

Based on the growth trend of the pre-experimental group, the third physical filtration was performed on the $19^{\text {th }}$ day of the experiment, $M$. aeruginosa was in

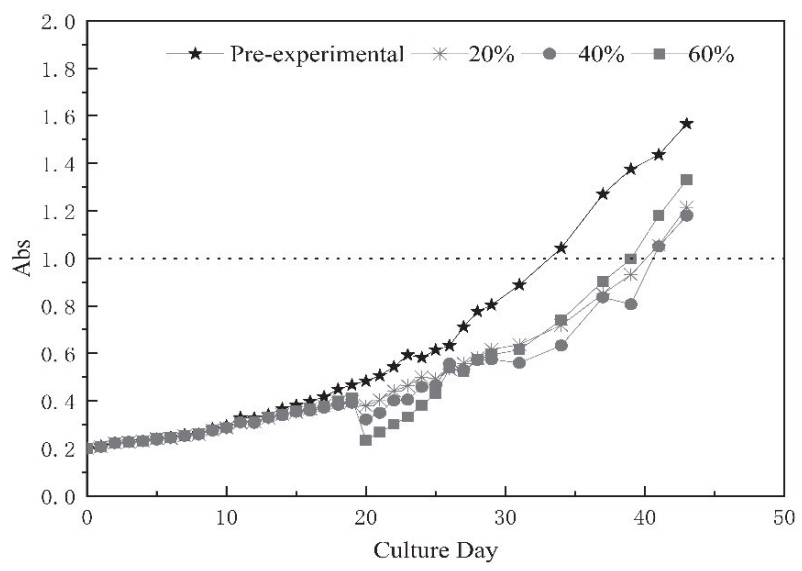

Fig. 7. The growth curve of Microcystis aeruginosa after physical filtration. the early stage of the logistic accelerated growth period. At this time, the proliferation rate of Microcystis aeruginosa was further accelerated and the absorbance value was about 0.45 . At this point, it had reached $\mathrm{k} / 4$ on the Logistic curve, which is an important stage for cyanobacteria to gradually form a competitive advantage.

After the third physical filtration, the absorbance of the filtration group decreased significantly. The absorbance of the filtration group was essentially equal to that of T20, T40, and T60 at 28 days. The filtration rate of Microcystis aeruginosa was as follows: T60>T40>T20. After the 28th day of culture, the absorbance of Microcystis aeruginosa under T60 control intensity was higher than that of T40 or T20. The growth pattern of Microcystis aeruginosa after this filtration was similar to that of the second filtration.

The $\mathrm{R}$ values of the pre-experimental group, T20, T40, and T60 were 0.98591, 0.98145, 0.94001, and 0.97262, respectively, after fitting with the Logistic model $(\mathrm{A} 2=2)$ (Fig. 8). The fitting curves showed that after the second physical filtration, the absorbance of the T20, T40, and T60 groups reached the maximum $\mathrm{K} / 2$ point at 39,41 , and 38 days, respectively. Different from the previous two filtration groups, it took less time for the absorbance of $M$. aeruginosa to reach $\mathrm{K}$ value after filtration. Moreover, with increased control intensity, Microcystis aeruginosa proliferation rate 

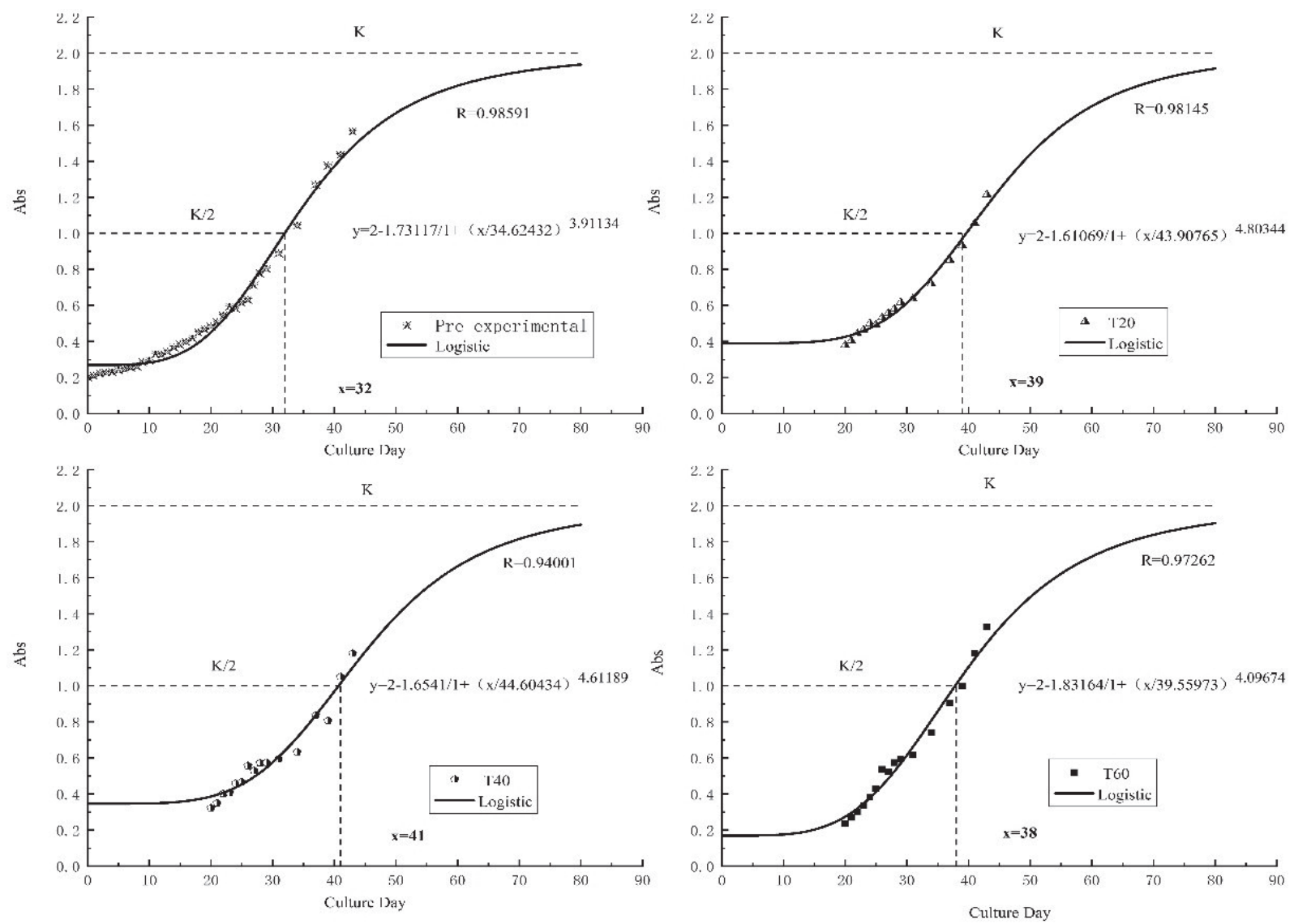

Fig. 8. Logistic fitting model of physical filtration.

also increased. The results demonstrated that physical filtration could inhibit the proliferation of Microcystis aeruginosa when it is growing rapidly, but the control effect was poor.

\section{Analysis of Physical Filtration}

This stage of the rapid proliferation of Microcystis aeruginosa is very critical and a good indication for the rapid propagation of cyanobacteria blooms in the natural aquatic environment. Cyanobacteria populations develop their competitive advantage by levitating in the water at this stage. At this time, cyanobacteria can also make full use of nutrients and light and gradually reach their maximum growth rate potential. This is also the previous stage when cyanobacteria enter the floating accumulation stage and thus rupture. At this stage, the simulated filtration has guiding significance to explore the control method of the cyanobacteria populations. For practical engineering guidance, physical filtering at this stage has a certain effect, but the effect is lower than the previous two filtering steps as Microcystis aeruginosa concentration is higher at this time. Although the concentration of Microcystis aeruginosa was reduced to a certain extent after filtration, it can still proliferate at a faster rate after filtration. The results showed that the growth rate of Microcystis aeruginosa with high control intensity was higher than that with low control intensity. Therefore, if physical filtration is carried out at this stage, it is best to use low control intensity for filtration at a lower cost.

Filtering in the Middle and Later Stages
of the Logistic Accelerated Growth Period

The absorbance of Microcystis aeruginosa reached 0.6 on the $25^{\text {th }}$ day of the culture period, which was

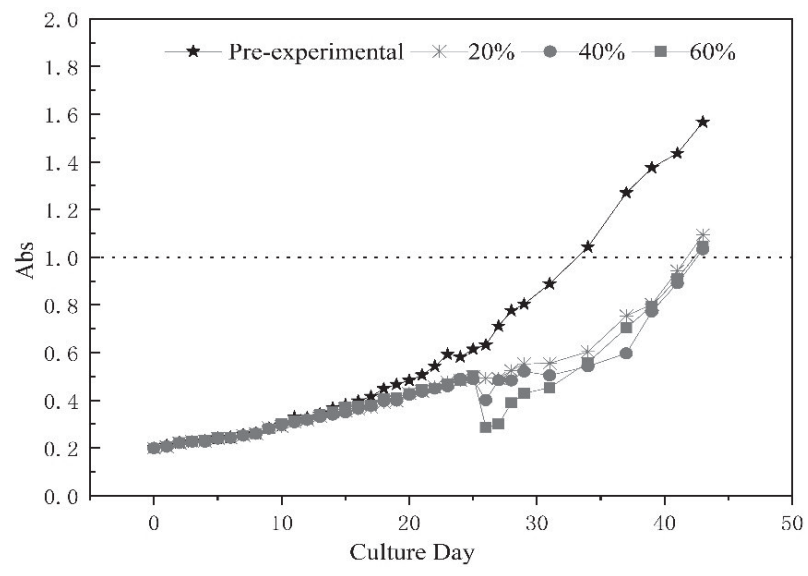

Fig. 9. The growth curve of Microcystis aeruginosa after the fourth physical filtration. 

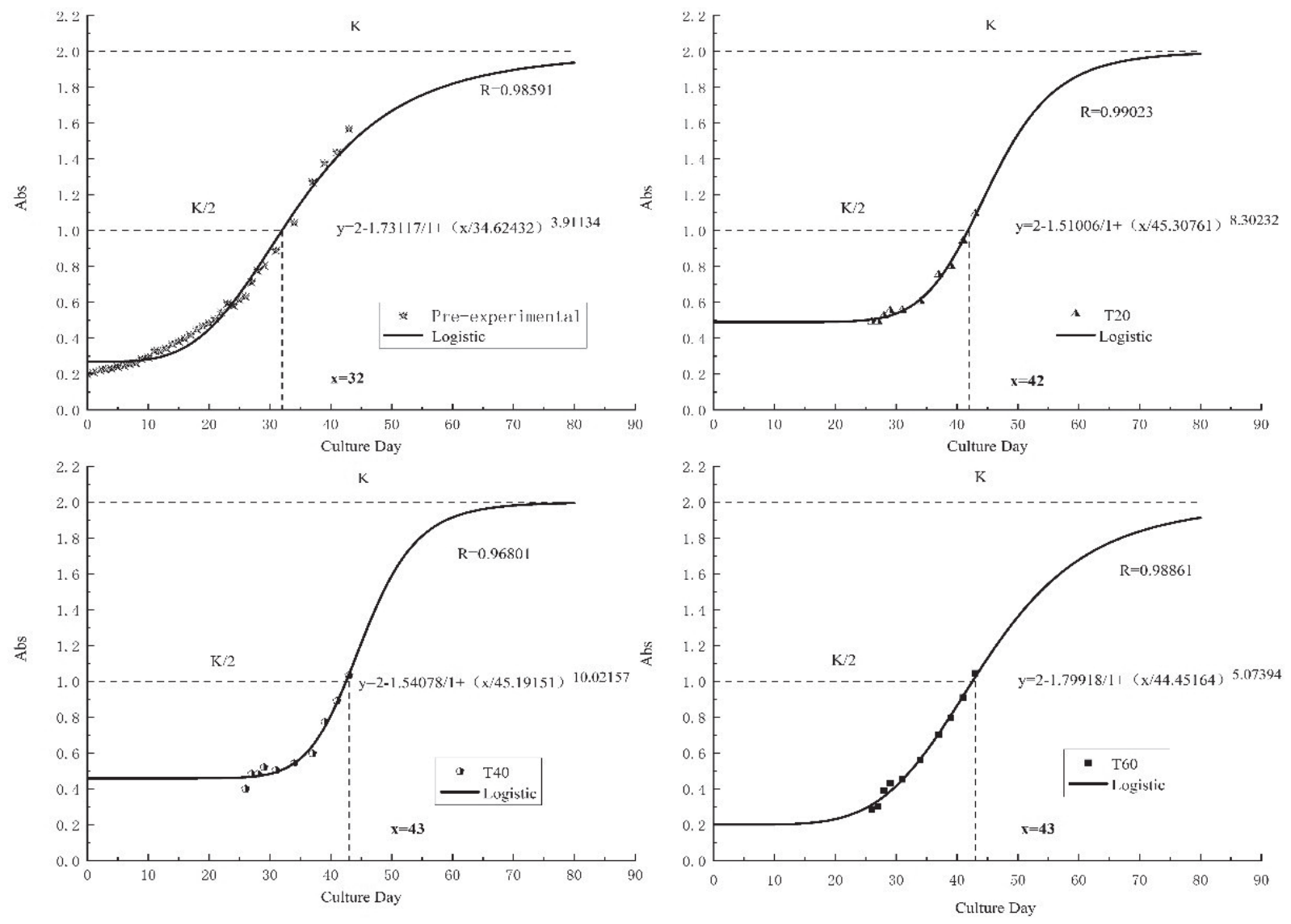

Fig. 10. Logistic fitting model of the fourth physical filtration.

close to the maximum growth rate point of this species $(\mathrm{K} / 2)$. In order to better explore the filtration rule of Microcystis aeruginosa near the fastest growth rate, the fourth physical filtration was performed on the $25^{\text {th }}$ day of the culture period.

After the fourth physical filtration, the absorbance of the filter group decreased to a certain extent, similar to that on the $40^{\text {th }}$ day (Fig. 9). Following filtration, the growth pattern was retained and the proliferation rate increased with higher control intensity.

The $\mathrm{R}$ values of the pre-experimental group, T20, T40, and T60 were 0.98591, 0.99023, 0.96801, and 0.98861 after fitting with the Logistic model $(\mathrm{A} 2=2)$ (Fig. 10). Although the follow-up monitoring time was short, there were only a few data points for Microcystis aeruginosa growth after physical filtration and the fitting degree was high. The growth trend of Microcystis aeruginosa after filtration can be simulated to some extent. After physical filtration, the absorbance of T20, T40, and T60 groups reached the maximum K/2 point at 42, 43, and 43 days, respectively (Fig. 9). The results showed that physical filtration could inhibit the growth of Microcystis aeruginosa at this stage.

\section{Analysis of Physical Filtration}

However, due to the short monitoring time after filtration and the high concentration of M. aeruginosa and a large amount of reduction, the culture reached $\mathrm{K} / 2$ and $\mathrm{K}$ points very quickly. In terms of practical projects, when the proliferation rate of cyanobacteria is fast and the concentration is high, physical filtration can quickly reduce the content of cyanobacteria in water, which has a certain effect. However, when the concentration of cyanobacteria is high, serious blooms have occurred in the natural aquatic environment. Although the filtration at this stage has certain inhibitory effects, it is equivalent to expanding the living environment of cyanobacteria after filtration. The concentration of cyanobacteria could be restored to the original concentration in a short time, and the harm caused by cyanobacteria bloom could not be effectively reduced. On the other hand, the simulation filtration experiment showed that the growth rate of Microcystis aeruginosa with high control intensity was higher than with low control intensity after filtration at this stage. When considering the cost, it is suggested to choose medium control intensity to salvage in a practical project.

\section{Conclusions}

This study performed a simulation filtration experiment where the dominant cyanobacteria $(M$. aeruginosa) were physically filtered four times. The growth feedback effect of $M$. aeruginosa after physical 
filtration was investigated. The research results are as follows:

1. The growth of $M$. aeruginosa during culture and after control was consistent with the Logistic growth model.

2. After Filtering in the early stages of the Logistic start period, $M$. aeruginosa growth was obviously inhibited. It is not suitable for the growth of $M$. aeruginosa in combination with the contents of nutrient elements and environmental factors in natural water, so the physical filtration effect is better in the early stage of cyanobacteria bloom. After Filtering in the later stages of the Logistic start period and in the early stage of the logistic accelerated growth period, as the filtration intensity increased so did the proliferation rate of $M$. aeruginosa. However, the growth of $M$. aeruginosa was inhibited during this period. Following cyanobacteria blooms, low control intensity can be selected to reduce the cost of salvage. After Filtering in the middle and later stages of the Logistic accelerated growth period, $M$. aeruginosa still reached $\mathrm{K} / 2$ and $\mathrm{K}$ points very quickly. Physical filtration in case of severe cyanobacteria bloom can reduce the bloom. However, cyanobacteria populations can recover to the original concentration in a short time, which cannot effectively reduce the harm caused by cyanobacteria bloom.

3. Based on the above results, in the actual cyanobacteria salvage project, if the dominant species is $M$. aeruginosa, it is suggested to select the control strength of about $20 \%$ at the beginning of the logistic growth. Physical salvage has good inhibitory effects on the growth of cyanobacteria and can effectively control the outbreak of cyanobacteria bloom after salvage.

\section{Abbreviations List}

GC-FID: Gas chromatography-flame ionization detector; GC-MS: Gas chromatography-mass spectrometry. N: Nitrogen; P: Phosphorus; K: Potassium; Ca: Calcium; Na: Sodium; Mg: Magnesium; S: sulfur; UV: Ultraviolet; MgSO4: Magnesium sulfate; PCA: Principal Component Analysis; r: correlation; ROS: Reactive Oxygen Species; $\mathrm{H}_{2} \mathrm{O}_{2}$ : hydrogen peroxide.

\section{Acknowledgments}

This study was supported by the National Natural Science Foundation of China (52070023) and the Changzhou Science and Technology Support Program (CE20205037). For the article writing and experiment, I would like to thank Mr. Xu for his inspiration and confidence in the idea and writing method, and $\mathrm{Mr}$. Zhao Fangxing and Miss. Pan Jie for their help in the experiment.

\section{Conflict of Interest}

The author states that there is no conflict of interest.

\section{References}

1. CAI Y.Y., TANG C.Y. A Kinetics Model for Predicting Microcystis Growth Based on the Synergistic Effect of Nitrogen and Phosphorus on the Growth of Microcystis densa (Cyanobacteria). Pol. J. Environ. Stud. 30 (2), 1067, 2021.

2. ZHU W., LI M., LUO Y.G., DAI X.X., GUO L.L., XIAO M., HUANG J.Y., TAN X. Vertical distribution of Microcystis colony size in Lake Taihu: Its role in algal blooms. J. Gt. Lakes Res. 40 (4), 949, 2014.

3. GRATTAN L.M., HOLOBAUGH S., MORRIS J.G. Harmful algal blooms and public health. Harmful Algae, 57 (PT. B), 2, 2016.

4. SHI K., ZHANG Y.L., QIN B.Q., ZHOU B.T. Remote sensing of cyanobacterial blooms in inland waters: present knowledge and future challenges. Science Bulletin, 64 (20), 76, 2019.

5. WANF G.L., LI J.S., ZHANG B., SHEN Q., ZHANG F.F. Monitoring cyanobacteria-dominant algal blooms in eutrophicated Taihu Lake in China with synthetic aperture radar images. Journal of Oceanology and Limnology, 33 (1), 139, 2015.

6. ZHANG L., LIU S.T., LIU T., YU C. Control effect of periodic variation on the growth of harmful algal bloom causative species. Commun. Nonlinear Sci. Numer. Simul. 54, 185, 2018.

7. LEKKI J., DEUTSCH E., SAYERS M., BOSSE K., ANDERSON R., TOKARS R., SAWTELL R. Determining remote sensing spatial resolution requirements for the monitoring of harmful algal blooms in the Great Lakes. J. Gt. Lakes Res. 45 (3), 434, 2019.

8. YANG L.Y., YANG X.Y., REN L.M., QIAN X., XIAO L. Mechanism and control strategy of cyanobacterial bloom in Lake Taihu. Journal of Lake Sciences, 31 (1), 18, 2019.

9. MAEKELAE S., HUITU E., TULONEN T., OJALA A. Control mechanisms of cyanobacterial blooms in lakes of varying trophic status and morphometry. International Association of Theoretical \& Applied Limnoloy, 29 (2), 953, 2005.

10. PAL M., YESANKAR P.J., DWIVEDI A., QURESHI A. Biotic control of harmful algal blooms (HABs): A brief review. J. Environ. Manage., 268, 110687, 2020.

11. ZHOU B.B., WANG G.X., YANG F., XIA M.F. Effective scooping out blue algae on growth of Microcystis aeruginosa. Journal of ecology and rural environment, 28 (3), 260, 2012.

12. PARK S.C., MOON J.C., KIM N.H., KIM E.J., JEONG J.E., NELSON A.D.L., JO B.H., JANG M.K., LEE J.R. Algicidal effect of hybrid peptides as potential inhibitors of harmful algal blooms. Biotechnol. Lett. 38, 847, 2016.

13. ECKERSLEY E., BERGER B.W. An engineered polysaccharide lyase to combat harmful algal blooms. Biochem. Eng. J. 132, 225, 2018.

14. SOUKAINA Z., FATIMA E.K., BRAHIM O., VITOR V. Seaweed Bioactive Compounds against Pathogens and Microalgae: Potential Uses on Pharmacology and Harmful Algae Bloom Control. Marine Drugs, 16 (2), 55, 2018. 
15. HENDERSON R., PARSONS S.A., JEFFERSON B. The impact of algal properties and pre-oxidation on solid-liquid separation of algae. Water Res. 42 (9), 1827, 2008.

16. LIU C., SHEN Q.S., ZHOU Q.L., FAN C.X., SHAO S.G. Precontrol of algae-induced black blooms through sediment dredging at appropriate depth in a typical eutrophic shallow lake. Ecol. Eng. 77, 139, 2015.

17. ZHANG J.Q., XIE Z.C., JIANG X.M., WANG Z. Control of Cyanobacterial Blooms via SynergisticEffects of Pulmonates and Submerged Plants. Clean, 43 (3), 330, 2015.

18. GAMEZ T.E., BENTON L., MANNING S.R. Observations of two reservoirs during a drought in central Texas, USA: Strategies for detecting harmful algal blooms. Ecol. Indic. 104 (SEP.), 588, 2019.

19. CHEN L.T., ZUO J., Tao S.Y., DAI G.F., SONG L.R., GAN N.Q. Progress in Control of Cyanobacteria by Microorganism. Journal of Wuhan University (Natural Science Edition), 65 (4), 401, 2019.

20. CHEN B.F., FENG M.H., SHANG L.X., KE F., WANG Y.R., LI Y. The release characteristics of algaederived pollutants in different salvage intensities after cyanobacteria accumulation. Acta scientiae circumstantiae, 36 (11), 4077, 2016.

21. LI X., CONG H.B. Study on the treatment process of cyanobacteria pulp and algae water separation wastewater from lake fishing. Technology of Water Treatment, 46 (2), 124, 2020.

22. VARATHAN N., WIJEKOON P. Logistic Liu Estimator under stochastic linear restrictions. Stat. Pap. 60 (4), 1, 2019.
23. TAPIA A., LEIVA V., GALEA M., WERNECK R. On a logistic regression model with random intercept: diagnostic analytics, simulation and biological application. J. Stat. Comput. Simul. 90 (4), 2354, 2020.

24. BAKER R.E., RST G. Global Dynamics of a Novel Delayed Logistic Equation Arising from Cell Biology. J. Nonlinear Sci. 30 (1), 397, 2020.

25. ZHOU L.R., BI Y.H., JIANG L.H., WANG Z.Q., CHEN W.Q. Effect of Black Wattle (Acacia mearnsii) Extract on Blue-Green Algal Bloom Control and Plankton Structure Optimization: A Field Mesocosm Experiment. Water Environ. Res. 84 (12), 2133, 2012.

26. QIU X.Y., XIE G.N. New ideas for salvaging cyanobacteria. Nonferrous Metal Design, 45 (4), 109, 2018.

27. LI L., ZHOU D.W., SHENG L.X. Allometric relationship between mean component biomass and density during the course of self-thinning for Fagopyrum esculentum populations. Acta Ecologica Sinica, 32 (13), 3987, 2012.

28. VOSPERNIK S., STERBA H. Do competition-density rule and self-thinning rule agree?. Ann. For. Sci. 72 (3), 379, 2015.

29. LIE Z.Y., XUE L. Density effect and self-thinning in Eucalyptus urophylla stands. J. For. Res. 30 (2), 153, 2019.

30. WU D.X., ZHANG T., BAI Y.F., WANG G., WANG G.X. Mechanism and universality of Yoda's $-3 / 2$ law of selfthinning in plant population. Chinese Journal Applied Ecology, 13 (9) 1081, 2002. 